\title{
Rursus
}

Ruscus Poiétique, réception et réécriture des textes antiques

$4 \mid 2009$

Erotica

\section{L'amour romanesque entre idéal et parodie : les romanciers grecs et Pétrone}

Aldo Setaioli

\section{OpenEdition}

Journals

Édition électronique

URL : http://journals.openedition.org/rursus/295

DOI : 10.4000/rursus.295

ISSN : 1951-669X

Éditeur

Université Nice-Sophia Antipolis

Référence électronique

Aldo Setaioli, «L'amour romanesque entre idéal et parodie : les romanciers grecs et Pétrone », Rursus [En ligne], 4 | 2009, mis en ligne le 01 février 2009, consulté le 19 avril 2019. URL : http:// journals.openedition.org/rursus/295; DOI : 10.4000/rursus.295

Ce document a été généré automatiquement le 19 avril 2019

Rursus 


\title{
L'amour romanesque entre idéal et parodie : les romanciers grecs et Pétrone
}

\author{
Aldo Setaioli
}

Bien que beaucoup de critiques s'accordent pour penser que les Satyrica de Pétrone sont une œuvre dépourvue de parallèles précis dans l'ensemble de la tradition littéraire de l'Antiquité ${ }^{1}$ une grande quantité de travail et d'ingéniosité a été consacrée à établir ses rapports avec la littérature précédente, en particulier avec le genre littéraire spécifique auquel les savants tendent à l'associer, celui qui est traditionnellement défini comme 'roman', même si les Anciens n'ont pas eu de terme particulier pour désigner le type d'écrits auquel pensent les modernes lorsqu'ils utilisent ce terme.

2 Il est vrai que dans les dernières décennies un certain nombre de découvertes papyrologiques bienvenues ont contribué à rompre l'isolement des Satyrica dans le panorama de ce que nous pouvons appeler la littérature narrative antique. Les fragments des Phoinikika de Lollianus², par exemple, non seulement nous offrent un exemple d'œuvre narrative assez différente des romans d'amour idéalisé que la tradition manuscrite nous a transmis dans leur intégralité, mais ils contiennent un certain nombre de situations comparables à plusieurs de celles que l'on rencontre chez Pétrone: une espèce d'orgie cultuelle, une scène de cannibalisme rituel (qui apparaît aussi chez Achille Tatius $^{3}$ ), et même des détails apparemment mineurs, comme les visages peints ${ }^{4}$. Une autre découverte importante, la publication d'un fragment de ce que l'on appelle le roman d' Iolaos, fut aussitôt saluée comme la découverte d'un "Satyricon grec", quoique avec l'addition louable d'un point d'interrogation ${ }^{5}$. Ce fragment non seulement contient une narration qui décrit une ambiance et une situation pouvant rappeler celles des Satyrica, mais il présente aussi un discours métrique en vers sotadiques prononcé par un personnage désigné comme gallus, et donc assez proche du cinaedus qui récite des vers sotadiques dans un épisode de Pétrone ${ }^{6}$. Un fragment narratif postérieur - connu sous le nom de Tinouphis ${ }^{7}$-, et comportant une section métrique a été publié par la suite, mais il est peut-être prématuré de considérer la forme prosimétrique des Satyrica comme 
l'héritage d'une forme préexistante dans la littérature narrative grecque, sans aucun rapport avec la tradition de la satire ménippée acclimatée à Rome par l'illustre prédécesseur qu'est Varron ${ }^{8}$.

3 Même avant ces récentes découvertes, il y avait des indices de l'existence de formes narratives bien différentes des romans d'amour idéalisé grecs; non seulement les œuvres que l'on connait sous le nom de fabulae Milesiae, dont les rapports avec ce que nous appelons le roman sont controversés, mais aussi la mention, dans la Souda, des Rhodiaka de Philippe d'Amphipolis comme une œuvre extrêmement obscène' ${ }^{9}$, et, bien avant, d'un auteur du nom d'Eubius et d'une œuvre intitulée Sybaritica, dans le long catalogue qui figure au livre II des Tristes d'Ovide ${ }^{10}$.

4 I. Parmi les œuvres narratives qui nous sont parvenues, les deux versions de l'histoire de l'âne - la version grecque du Pseudo-Lucien et la version latine d'Apulée - semblent appartenir à un type différent de celui représenté par les cinq romans d'amour transmis par la tradition manuscrite : ceux de Chariton, d'Achille Tatius, de Xénophon d'Éphèse, de Longus et d'Héliodore ; mais, malgré quelques caractères communs, ils ne semblent pas ressembler non plus exactement aux Satyrica; et, malheureusement, nous ne savons quasiment rien de l'autre roman qu'aurait écrit Apulée, intitulé Hermagoras ${ }^{11}$.

L'histoire de l'âne démontre que des œuvres narratives de caractère comique existaient en grec ; mais même sans elle, il serait clair que le roman d'amour n'était pas le seul type de littérature narrative grecque ${ }^{12}$. L'Histoire véritable de Lucien démontre suffisamment l'existence de la parodie d'un certain genre narratif, celui des voyages. Ce n'est ni le moment ni le lieu ici de discuter l'épineux problème de l'origine et de l'évolution du roman grec, problème qui a été au centre du débat savant qui a suivi la publication du livre d'Erwin Rohde ${ }^{13}$ - lequel demeure un travail fondamental, même si les découvertes successives ont démontré la fausseté de la chronologie proposée par Rohde. L'objet de notre enquête, ce sont plutôt les rapports entre les Satyrica de Pétrone et les romans érotiques grecs, représentés à nos yeux par les cinq auteurs que nous venons de rappeler.

Présupposer l'existence de tels rapports paraît justifié par le fait qu'un auteur antique rapproche explicitement les œuvres des deux romanciers latins - Pétrone et Apulée - du roman d'amour. Dans un passage célèbre de son commentaire au Somnium Scipionis de Cicéron, Macrobe caractérise les œuvres narratives de ces auteurs en leur appliquant une définition qui, de toute évidence, avait été forgée par référence aux romans érotiques grecs, comme le démontre un parallèle précis figurant dans une lettre de l'empereur Julien l'Apostat. Macrobe appelle les histoires racontées par Pétrone et Apulée « intrigues pleines d'aventures imaginaires d'amoureux »; de même Julien emploie les expressions " histoires imaginaires » et "intrigue amoureuse ${ }^{14}$, en référence évidente aux romans grecs. Il est évident que l'écrivain latin, à la différence des théories de divers savants modernes, n'hésitait pas à appliquer aux romanciers romains $-\mathrm{y}$ compris Apulée, dans les Métamorphoses duquel n'apparaît aucun couple d'amoureux- la définition caractérisant le type d'écriture narrative qui prévalait à son époque : le roman d'amour. Cela montre qu'il considérait toutes les œuvres narratives comme relevant d'un unique genre littéraire - une position adoptée aujourd'hui par de nombreux savants ${ }^{15}$. Il sera donc justifié de rechercher des parallèles avec l'œuvre pétronienne dans toutes les œuvres narratives de l'antiquité. Mais, une fois établi qu'il est licite de s'attendre à des parallèles et à des ressemblances entre les Satyrica et le roman d'amour, le type et le caractère de tels parallèles reste à définir et à évaluer. 
7 À la fin du XIX siècle, Richard Heinze vit dans les Satyrica une parodie continue des romans érotiques grecs qui tendait à dégrader et désacraliser une série de thèmes et de motifs utilisés de façon tout à fait sérieuse par les romanciers grecs ${ }^{16}$. Son analyse est pénétrante et convaincante, et, presque à la fin, il suppose même l'existence d'un roman parodique grec précédant Pétrone ${ }^{17}$. La position de Heinze a été adoptée par plusieurs savants, quoique parfois avec des réserves et des restrictions ${ }^{18}$; mais elle a été repoussée par bien d'autres.

8 Une des réserves les plus sérieuses concerne la chronologie du matériel littéraire à confronter avec ce qui reste des Satyrica de Pétrone, dans le but de vérifier l'attitude parodique attribuée à ce dernier. La majeure partie des romans grecs qui nous sont parvenus est sans aucun doute postérieure à l'œuvre de Pétrone, même si les papyri démontrent que Chariton a vécu à une époque guère plus tardive ${ }^{19}$, et que l'œuvre connue sous le nom de Roman de Ninus, dont les fragments prouvent que l'élément érotique n'était pas absent de l'intrigue, est probablement antérieure à Pétrone ${ }^{20}$. Les autres arguments allégués pour une datation plus haute du roman en général et du roman d'amour en particulier sont trop vagues pour qu'on les considère comme définitifs ${ }^{21}$

Même si un certain nombre de parodies des thèmes courants dans le roman d'amour ont déjà été signalées chez Pétrone ${ }^{22}$, la liste est bien loin d'être complète et, surtout, aucun savant, à ma connaissance, ne s'est livré à un examen détaillé de chacune de ces reprises parodiques. C'est précisément cela que nous nous proposons de faire dans le cours de cette recherche ; si, à la fin de notre examen, il apparaît clairement que les mêmes motifs qui ceux qui sont utilisés sérieusement dans les romans érotiques grecs font l'objet chez Pétrone d'une déformation ridicule ou irrévérencieuse, nous ne pourrons éviter de nous demander si cela tient au hasard ou à des raisons objectives ${ }^{23}$. Et si le phénomène se montre régulier et constant, nous serons contraints d'écarter l'hypothèse de coïncidences accidentelles, et nous devrons aller plus loin, en nous demandant lequel, de l'usage sérieux ou de la parodie, a le plus de chance d'avoir précédé l'autre ${ }^{24}$. Et comme, à mon avis, on ne peut avoir de doute sur la réponse à donner à cette question, nous devrons souscrire à la position de Heinze, qui soutenait l'existence d'un roman d'amour pleinement développé antérieurement à Pétrone, cela avant même que l'on ne disposât de la documentation papyrologique pour étayer son affirmation, et même si sa confrontation entre Pétrone et les romans grecs était tout sauf exhaustive. À ce propos, on ne considèrera peut-être pas comme une pure coïncidence le fait que, même dans les romans érotiques grecs qui nous sont parvenus, composés sans l'ombre d'un doute à l'époque romaine, n'apparaitt rigoureusement aucune allusion à l'existence de l'empire romain ${ }^{25}$.

Préalablement, cependant, nous devons rappeler que nous possédons seulement une petite partie des Satyrica, et - ce qui est encore plus important - que l'œuvre de Pétrone est bien plus qu'une simple parodie, qu'elle est plutôt un chef d'œuvre littéraire absolu, qui, comme nous l'avons remarqué plus haut, n'a aucun parallèle précis dans l'antiquité. Il suffit de penser à la Cena Trimalchionis, même si des banquets ont été occasionnellement décrits dans d'autres romans ${ }^{26}$. La parodie et la désacralisation sont des éléments fondamentaux de cette œuvre unique, mais cette affirmation devra être soigneusement nuancée. L'influence de la satire est évidente dans les Satyrica ${ }^{27}$, mais des allusions à des genres littéraires divers sont omniprésents et indubitables chez Pétrone; elles consistent souvent dans la parodie et la désacralisation des formes littéraires les plus hautes, comme l'épopée et la tragédie. 
11 Certains savants ont cherché à réduire la portée de la parodie du roman d'amour dans les Satyrica en insistant sur ce niveau différent de parodie ${ }^{28}$; d'autres ont fait remarquer que la structure de l'œuvre de Pétrone ne permettait la présence que d'une gamme réduite de situations comparables à celles qui se rencontrent dans les romans d'amour conventionnels ${ }^{29}$. Les Satyrica sont certainement plus libres et de plus large portée, et ne sont pas liés aux modèles structurels qui gouvernent ces derniers - par exemple, Encolpe et Giton ne restent séparés que pendant un petit nombre de chapitres ${ }^{30}$. Cependant, comme nous le verrons, la parodie du genre narratif est clairement reconnaissable et en outre la dégradation des modèles de la grande littérature en est inséparable.

En premier lieu, dans les romans d'amour aussi nous nous trouvons devant le croisement de divers genres littéraires, même si le résultat que visent ainsi les auteurs grecs est le raffinement littéraire, tandis que Pétrone, bien que tout aussi raffiné, voire davantage, cherche la parodie et la désacralisation. Il est facile d'observer que l'habitude de Chariton d'introduire des vers homériques au sein de sa prose, ou l'utilisation d'expressions virgiliennes empruntées à l'épisode de Didon dans l'Histoire d'Apollonios ${ }^{31}$, qui dans ces œuvres ont un caractère tout à fait sérieux, trouvent un parallèle joyeux et parodique dans l'usage que fait Pétrone d'Homère et de Virgile, lorsqu'il inclut des allusions irrévérencieuses au même épisode de l'Énéide ${ }^{32}$. Il est évident que le but de Pétrone est de tourner en ridicule non Virgile, le Romanus Vergilius qu'il admire ${ }^{33}$, mais plutôt l'usage de la grande poésie dans les écrits du type de ceux que avons mentionnés. Il en va de même en ce qui concerne Homère: Pétrone base une composition poétique entière, dans l'épisode de Circé des Satyrica ${ }^{34}$, sur un épisode du livre XIV de l'Iliade (la scène d'amour entre Zeus et Héra sur le mont Ida) ; dans une situation analogue, Héliodore s'y réfère à son tour ${ }^{35}$. Les deux passages décrivent les tentatives d'une rivale de l'héroïne pour séduire le héros du roman; mais alors que chez Héliodore l'entremetteuse cybèle se réfère aux paroles de Zeus promettant à Héra de dissimuler leur amour même à la vue pénétrante du Soleil, Pétrone développe bien plus abondamment le motif ${ }^{36}$, même s'il n'omet pas l'allusion au secret qui était au premier plan chez Héliodore ${ }^{37}$. Mais il place sa variation poétique raffinée sur le thème homérique dans un contexte où Encolpe, dans son exaltation amoureuse, se met lui-même sur le même plan que Jupiter, voire au-dessus de lui - comme dans le poème blasphématoire qui précède ${ }^{38}-$, pour être ensuite frappé d'une impuissance sexuelle inattendue dans le dénouement ridicule. Et quand, peu après ${ }^{39}$ , Encolpe justifie son discours à sa mentula en se référant d'abord à celui d'Ulysse à son propre cœur ${ }^{40}$, puis en mentionnant les personnages tragiques qui s'adressent à leurs propres yeux comme si ceux-ci pouvaient entendre, il ne ridiculise pas seulement, et même pas principalement, l'Oedipe de Sophocle ${ }^{41}$, mais plutôt des écrivains comme Chariton et comme les auteurs de l'Histoire d'Apollonios et des fragments de Kalligoné, qui imitent la tragédie en mettant en scène des personnages qui, de façon parfaitement sérieuse, s'adressent à leurs propres yeux ${ }^{42}$. Il est donc évident que chez Pétrone la parodie des modèles élevés est imbriquée inextricablement avec celle des romans d'amour. Si dans les Satyrica elle paraît tout à fait envahissante, cela est dû principalement à la grande culture littéraire de l'auteur, largement supérieure, ou du moins bien mieux dominée et assimilée que dans la plupart des romans grecs que nous avons encore la possibilité de lire.

13 II. Comme nous l'avons déjà montré, il ne faut jamais perdre de vue le fait que ce que nous possédons des Satyrica n'est qu'une petite partie de l'œuvre originale de Pétrone. 
Toutefois, et malgré aussi la complexité littéraire bien plus grande que nous avons mise en évidence, un schéma parodique clair est aisément reconnaissable.

La différence la plus évidente, reconnue depuis $H_{e i n z e}{ }^{43}$, réside dans le couple d'amoureux, qui chez Pétrone est homosexuel: Encolpe et Giton. Certains savants refusent de voir une intention parodique dans ce détail, en soulignant que des thèmes pédérastiques apparaissent aussi dans les romans grecs ${ }^{44}$; mais il est facile de répondre que dans ces derniers ces thèmes sont limités aux personnages secondaires, tandis que le couple qui se trouve au centre de la trame narrative est invariablement hétérosexuel ${ }^{45}$. On a en outre observé que le motif récurrent de l'ekphrasis de représentations artistiques de scènes amoureuses, toujours hétérosexuelles dans les romans grecs ${ }^{46}$, se réfère exclusivement à des couples homosexuels chez Pétrone ${ }^{47}$. En outre, la présence simultanée chez lui des trois motifs qui apparaissent au début de l'œuvre d'Achille Tatius (ekphrasis, puissance de l'amour, explication de l'œuvre d'art donnée par un autre personnage) fait qu'il est difficile de croire que Pétrone n'avait pas en tête de façon précise un modèle romanesque. La même transposition est reconnaissable dans le cas d'un thème qui, pour être probablement né dans un contexte homoérotique et avoir été développé par des auteurs du niveau de Platon et de Callimaque, avait été transposé à l'amour hétérosexuel par les auteurs de romans, mais qui est ramené par Pétrone à la sphère homosexuelle dans une poésie d'un grand raffinement littéraire ${ }^{48}$ : la fusion et l'échange des âmes des amants à travers le baiser. Les exactes correspondances textuelles et conceptuelles entre la poésie de Pétrone et certains passages d'Achille Tatius sur le baiser hétérosexuel, entre homme et femme, que j'ai illustrées et discutées ailleurs ${ }^{49}$, font qu'il est difficile de penser que Pétrone - même si naturellement il se réfère à l'ensemble de la tradition du motif -ne se proposait pas une parodie intentionnelle du roman d'amour ${ }^{50}$ avec cette poésie, tout comme, plus tard, avec la description du baiser entre Encolpe et le jeune Endymion ${ }^{51}$.

16 Mais le sexe des deux amoureux n'est pas la seule, et peut-être pas non plus la plus grande différence entre le couple pétronien et ceux des romans grecs. Dans ces derniers, les héros masculins apparaissent parfois, selon notre goût et notre sensibilité, comme de simples marionnettes privées de vie, non seulement dans la mesure où ils appartiennent à des classes sociales supérieures à celle des protagonistes de Pétrone, mais, en outre, parce qu'ils sont invariablement nobles et vertueux. Encolpe au contraire à été souvent défini déjà comme un 'anti-héros' ; et cela peut nous autoriser à considérer le roman d'amour comme l' 'anti-modèle', ou du moins comme un anti-modèle de l'œuvre de Pétrone.

17 Encolpe est prêt à déclarer son amour, à Giton comme à Circé, même quand ceux-ci l'ont maltraité52, mais - à la différence des héros masculins des romans d'amour - il est toujours prêt par ailleurs à chercher de nouvelles aventures, non seulement avec Circé, mais aussi avec le garçon de Crotone ${ }^{53}$. Giton, de son côté, est lui aussi bien différent des héroïnes fidèles en amour des romans grecs. Non seulement il est prêt à profiter de la situation chaque fois que cela lui paraît possible ${ }^{54}$, mais il ne se montre jamais un partenaire loyal et de confiance. Le choix qu'il fait d'Ascylte à la place d'Encolpe se déroule dans une scène au comique très efficace - supérieur même au comique de la scène comparable du Roland furieux où Doralice, contre toute attente, choisit Mandricardo au lieu de Rodomonte ${ }^{55}$. Dans le détail, cet épisode humoristique entier rappelle une série de situations décrites de façon tout à fait sérieuse dans les romans d'amour, comme celle qui figure chez Chariton ${ }^{56}$, lorsque Callirhoé doit choisir entre Dionysios et Chéréas. Dionysios maudit Babylone, comme le fait Encolpe pour la Graeca urbs ${ }^{57}$; Callirhoé, au 
contraire, refuse pudiquement d'exprimer son choix, tandis que Giton, dans un retournement précis qui semble bien être intentionnel, n'hésite pas un instant à le faire savoii ${ }^{58}$. L'effet parodique est à son comble plus loin, lorsque Giton, en réponse à une question précise d'Encolpe, déclare ne pas avoir subi de violences de la part d'Ascylte ${ }^{59}$ - déclaration dont le clin d'oeil humoristique a échappé à Heinze, quand, avec une ingénuité égale à celle d'Encolpe, il interprète les paroles de Giton comme signifiant que celui-ci n'a pas eu de rapports avec Ascylte, c'est-à-dire de la façon même dont le jeune homme veut que son amoureux les comprenne ${ }^{60}$.

Ce n'est pas seulement le couple des amants qui chez Pétrone se comporte à l'inverse du couple des héros des romans érotiques ${ }^{61}$; cela est vrai aussi pour leurs compagnons d'aventure. L'ami fidèle est un personnage traditionnel du roman ${ }^{62}$; mais Ascylte est certainement bien différent de Polycharme chez Chariton, de Cnémon chez Héliodore, ou de Clinias chez Achille Tatius ; et quand un compagnon plus âgé, le poète Eumolpe, prend la place d'Ascylte, il se montre bien éloigné du personnage vénérable du vieux Calasiris dans le roman d'Héliodore.

Ces contrastes dans la représentation des personnages me semblent bien plus importants que la prétendue parodie du thème de la divinité persécutrice des romans grecs, reconnue dans le rôle que joue la figure divine licencieuse de Priape chez Pétrone ${ }^{63}$, à la suite de l'article fondamental de Klebs ${ }^{64}$. Aujourd'hui, cependant, le rôle central de Priape dans les Satyrica a été mis en doute par divers savants ${ }^{65}$.

III. Examinons maintenant un certain nombre de thèmes et motifs particuliers. Mon intention n'est pas de distinguer nettement entre scènes et épisodes typiques amplement développés d'un côté, et motifs apparaissant de façon plus sporadique et isolée, étant donné qu'il n'est pas rare que les premiers incluent un de ces derniers, voire davantage.

Considérons, par exemple, un épisode caractéristique de presque tous les romans: la tempête marine qui presque inévitablement comporte un naufrage. On a affirmé souvent que dans ce cas la description de Pétrone ne contient pas d'élément parodique ${ }^{66}$. Mais à part le fait que la tempête de Pétrone s'achève avec l'épisode absurde, encore qu'extrêmement ingénieux, d'Eumolpe qui refuse d'être sauvé avant d'avoir donné une conclusion satisfaisante au poème qu'il est en train d'écrire ${ }^{67}$, la description de Pétrone contient des allusions répétées à un thème apparenté qui semble avoir échappé aux critiques. Tant Héliodore qu'Achille Tatius se réfèrent à la chaloupe qui se trouvait à bord du navire. Chez Héliodore ${ }^{68}$, les pirates permettent aux marins phéniciens de quitter le navire sur la chaloupe, comme certains d'entre eux avaient déjà tenté de le faire ${ }^{69}$. D'un autre côté, chez Héliodore, le calme de la mer produit l'effet contraire à celui qu'il a chez Pétrone : non pas paix et joie, mais guerre et désespoir, parce qu'il permet aux pirates de rejoindre le navire. Chez Achille Tatius les passagers du navire se battent entre eux pour arriver à monter dans la chaloupe ${ }^{70}$. Chez les deux auteurs grecs cela se produit dans des circonstances tragiques. Chez Pétrone la chaloupe est utilisée à la fin de façon normale, lorsque Tryphène se sauve du naufrage grâce à elle ${ }^{71}$, mais auparavant elle joue un rôle important dans les délibérations humoristiques destinées à imaginer le moyen de fuir la colère de Lichas ${ }^{72}$. Encolpe propose de l'utiliser pour quitter le navire, mais nous apprenons qu'elle est surveillée nuit et jour.

Nous pourrions citer un certain nombre d'autres motifs communs à Pétrone et aux romans grecs qui semblent utilisés sans intention parodique dans les Satyrica: les marques sur les murs et les colonnes afin de se retrouver, comme chez Héliodore ${ }^{73}$, ou de retrouver son chemin, comme chez Pétrone ${ }^{74}$; également la description de la capture des 
oiseaux à la glu - une excuse des amants pour se revoir chez Longus ${ }^{75}$ et une scène d'un grand effet artistique chez Pétrone ${ }^{76}$, qui omet l'évocation prosaïque du moment où les oiseaux sont tués et plumés (moment décrit soigneusement par Longus), pour s'attarder au contraire sur le tableau des plumes transportées par le vent et tournoyant dans la mer.

Les honneurs funèbres rendus à l'ennemi d'Encolpe, Lichas ${ }^{77}$, peuvent être confrontés avec ceux attribués à Dorcon, le rival de Daphnis dans le roman de Longus ${ }^{78}$. Le locus amoenus décrit par Achille Tatius ${ }^{79}$ rappelle une description poétique de Pétrone ${ }^{80}$. Il s'agit naturellement d'un thème commun à divers genres littéraires, mais les ressemblances entre les deux romanciers sont bien précises. Même si dans la poésie pétronienne décrivant le locus amoenus on ne perçoit pas de parodie, il ne faut pas oublier le motif humoristique de l'impuissance d'Encolpe qui parcourt le contexte tout entier. Un autre motif courant est la description de la beauté d'un personnage féminin, même si ce n'est pas non plus un thème limité exclusivement au roman. Si l'on confronte les descriptions de Leucippé ${ }^{81}$ et de Mélité ${ }^{82}$ chez Achille Tatius, on ne peut manquer d'apercevoir dans les deux cas un schéma préétabli : toutes deux ont les yeux brillants, les cheveux blonds, le teint blanc et les joues roses. Quand nous lisons la description de Circé chez Pétrone, nous trouvons aussi des yeux brillants et un teint blanc comme le marbre ; en outre elle a aussi des cheveux bouclés, comme Leucippé, et comme elle, elle est comparée à une œuvre d'art ${ }^{83}$. D'un autre côté, lorsque Encolpe déclare qu'à la vue de Circé sa beauté lui inspire du dédain pour celle de son ancien amour Doris ${ }^{84}$, cette affirmation trouve un parallèle parfait dans les paroles de Clitophon chez Achille Tatius : il croyait Calligoné belle tant qu'il n'avait pas vu Leucippés5.

Un autre thème qui ne semble pas parodié chez Pétrone - même si certains y voient des éléments parodiques ${ }^{86}$ - est la scène dans laquelle Giton et Encolpe se préparent à mourir dans les bras l'un de l'autre au milieu de la tempête en furie ${ }^{87}$. Le couple d'amants pris dans un naufrage est un motif topique dans le roman ${ }^{88}$, et le désir de mourir ensemble est un autre thème courant dans ce genre littéraire ${ }^{89}$. Comme nous l'avons vu, Giton est souvent rusé et déloyal, mais ici le péril mortel est imminent et réel, et il ne laisse pas de place à la simulation.

Il en est bien autrement dans les descriptions des tentatives théâtrales de suicide mises en œuvre par les deux partenaires. L'intention ou la tentative de se tuer est peut-être le thème qui entre tous revient à satiété dans les romans grecs, surtout quand un des deux amoureux croit que l'autre est mort ou perdu pour toujours. L'idée fondamentale du lien à la vie à la mort entre les amants trouve peut-être son expression la plus prégnante dans les paroles de Chariclée chez Héliodore: «Le destin fixé pour moi par les puissances divines est de vivre avec cet homme aussi longtemps qu'il vit et de mourir avec lui quand il mourra $»^{90}$. Il s'agit d'une idée très facile à parodier, dans l'Antiquité comme à l'époque moderne. Dans le Mikado de Gilbert et Sullivan, par exemple, Yum-Yum chante : «When your time has come to perish, / then the maiden whom you cherish / must be slaughtered too»; et elle ajoute : «With a passion that's intense / we worship and adore, / but the laws of common sense / we oughtn't to ignore». Chez Pétrone Encolpe décide de se pendre quand il croit que Giton l'a définitivement abandonné ${ }^{91}$. Cela nous rappelle l'épisode déjà mentionné du roman de Chariton, lorsque Callirhoé doit choisir entre Chéréas et Dionysios. Chéréas, désespéré, noue une corde pour se pendre ${ }^{92}$. Le début du discours qu'il prononce ${ }^{93}$ ressemble de près aux premiers mots d'Encolpe abandonné par Giton ${ }^{94}$; lui comme Chéréas déplorent d'avoir survécu à de précédentes aventures pour se trouver abandonnés par la personne aimée; mais bien vite le ton de leur discours diverge 
radicalement : Chéréas renonce noblement à Callirhoé, tandis qu'Encolpe maudit Ascylte et Giton avec les injures sexuelles les plus crues. Naturellement ni Chéréas ni Encolpe ne se tuent vraiment - le héros d'un roman ne peut le faire, dans la mesure où l'histoire doit continuer. Le seul véritable suicide amoureux se rencontre dans les Métamorphoses d'Apulée ${ }^{95}$, où Charité se tue sur la tombe de son époux Tlépolème; mais les Métamorphoses ne présentent pas de couple d'amoureux au centre de l'intrigue, et Charité n'est pas l'héroïne du roman.

Encolpe tente à nouveau de se tuer peu après, alors qu'il croit que Giton s'est suicidé pour de bon ${ }^{96}$. Sa précédente tentative de pendaison avait été interrompue par le retour à l'improviste de Giton; mais le garçon, en le voyant prêt à mourir, veut le précéder et se frappe avec un rasoir appartenant à l'esclave d'Eumolpe ${ }^{97}$. Il s'agit d'un rasoir qui se révèlera émoussé, car il est utilisé par les apprentis barbiers pour apprendre le métier. On peut discuter à l'infini sur la sincérité du geste de Giton, mais nous ne saurons jamais s'il était au courant ou non du fait que le rasoir était émoussé. Il est possible de mettre en doute sa bonne foi, étant donné que peu auparavant il avait affirmé avoir cherché en vain une épée pour se suicider dans la demeure d'Ascylte ${ }^{98}$ - et nous savons de façon sûre qu'Ascylte en possédait une ${ }^{99}$. Ce qui est certain, cependant, c'est qu'après s'être frappé Giton feint théâtralement d'être mort en se jetant à terre ${ }^{100}$ : une mimica mors, comme le dit Encolpe un peu plus tard avec justesse ${ }^{101}$, mais sur le moment cette 'mort' le pousse à une seconde tentative de suicide, avec le même rasoir. Le thème de l'arme inoffensive se trouve chez Achille Tatius ${ }^{102}$ : un poignard dont la lame rentre dans le manche, avec lequel Ménélas feint de tuer Leucippé : c'est aussi une mimica mors, dans la mesure où l'auteur nous dit que ce poignard était utilisé par les acteurs sur la scène ${ }^{103}$.

Les tentatives théâtrales de suicide continuent à bord du navire de Lichas, une fois qu'Encolpe et Giton ont été reconnus ${ }^{104}$. Giton menace de se castrer - mais avec le rasoir émoussé utilisé précédemment ; encore un geste théâtral appelé à juste titre tragoedia ; et Encolpe se met sur la gorge un couteau de barbier, même si cette fois il n'a pas l'intention de mettre à exécution sa menace, pas plus que Giton. Le rôle joué par les deux amants pétroniens - sans parler de la nature incongrue de leurs armes - ne peut qu'apparaître comme une parodie caricaturale de situations romanesques comme celle qui apparaît au début des Ethiopiques d'Héliodore, lorsque Chariclée pointe une épée sur son sein en menaçant de se tuer si elle doit être séparée de Théagène ${ }^{105}$. Pétrone a exploité de la façon la plus habile toutes les suggestions offertes par un thème romanesque répandu pour monter un épisode réjouissant, dont la valeur artistique va bien au-delà de la simple parodie, même si elle est à la base du processus littéraire mis en œuvre.

Nous avons vu que Pétrone fait référence à diverses reprises au théâtre en donnant un tour humoristique au thème de la tentative de suicide des amants. La composante théâtrale occupe une place éminente chez Pétrone, comme l'a magistralement montré Costas Panayotakis ${ }^{106}$; et cela est vrai aussi pour les romans d'amour sérieux ${ }^{107}$. Dans le passage dont nous avons parlé au début, Macrobe associe les romans d'amour, pour lesquels, comme nous l'avons vu, il prend pour exemples inattendus Pétrone et Apulée, aux comédies de Ménandre ${ }^{108}$. Les passages dans lesquels les romanciers grecs se réfèrent à leur propre récit au moyen de termes empruntés au théâtre sont innombrables chez tous les auteurs, et particulièrement chez Héliodore ${ }^{109}$. Cela arrive parfois aussi chez Pétrone ${ }^{110}$, mais le plus souvent dans les Satyrica les termes théâtraux sont utilisés pour indiquer la fiction et l'illusion : la mimica mors ${ }^{111}$ que nous avons déjà mentionnée, les mimicae artes visant à tromper Lichas ${ }^{112}$, ou le mimus qu'Eumolpe et ses compagnons se 
préparent à mettre en scène pour tromper les habitants de Crotone ${ }^{113}$, et qui auparavant ont été appelés tragoedia ${ }^{114}$, tout comme celle jouée par Giton avec le rasoir émoussée ${ }^{115}$. Il n'y a pas de différence, à ce qu'il semble, entre la tragédie pleine de dignité et le mime populaire. Parfois aussi les romans grecs font allusion au théâtre comme à un jeu visant à tromper ${ }^{116}$; mais il ne faut probablement pas voir une pure coïncidence dans le fait que, alors que dans les romans sérieux cet emploi est beaucoup plus rare que l'usage de termes théâtraux se référant à la conduite de la narration, chez Pétrone en revanche la situation apparaît exactement inverse.

Un autre thème typique, qui remonte jusqu'à l'Odyssée ${ }^{117}$, est le makarismos, c'est-à-dire la proclamation du bonheur du partenaire ou des parents du héros ou de l'héroïne. Cela se trouve non seulement dans les romans d'amour ${ }^{118}$, mais aussi chez Apulée, encore qu'adapté à l'atmosphère religieuse du dernier livre des Métamorphoses ${ }^{119}$. Une claire parodie de ce thème apparaît dans les avances d'Eumolpe à Giton ${ }^{120}$, dont la mère est proclamée heureuse en raison de la beauté et de la vertu de son fils. Le vieux poète ajoute qu'Encolpe ne sera pas jaloux, du moment qu'il en aime un autre - provoquant naturellement la colère de ce dernier. Il vaut peut-être la peine de noter que tandis que dans les romans sérieux ce sont les deux parents ${ }^{121}$ ou le père ${ }^{122}$ qui sont proclamés heureux, Eumolpe en revanche fait référence à la mère, comme dans un passage évangélique bien connu ${ }^{123}$.

Un autre exemple d'emploi humoristique d'un thème au moins aussi ancien que l'Archiloque de Cologne ${ }^{124}$, mais qui fut repris aussi par le roman, concerne le motif de l'amour couvert seulement d'un manteau. Chez Longus, Daphnis et Chloé sont couchés nus sous la même peau de chèvre ${ }^{125}$. Il est possible que la scène contienne une légère parodie également chez le romancier grec, étant donné que Daphnis contrôle sa passion, de telle sorte que la scène d'amour attendue n'a pas lieu; mais la parodie est beaucoup plus évidente lorsqu'Ascylte soulève le manteau sous lequel Encolpe et Giton sont occupés à faire l'amour, et qu'il demande : "Que faisais-tu, très saint frère? Vous faites tente commune sous ton vêtement?» ${ }^{126}$ Le même thème apparaît à nouveau, mais cette fois sublimé par l'imminence de la mort, dans la scène déjà signalée où Giton se glisse sous la tunique d'Encolpe pour mourir avec lui dans le naufrage ${ }^{127}$.

31 Quand chez Héliodore le roi d'Éthiopie ordonne de prendre soin de Chariclée et de Théagène et de les nourrir abondamment en vue de leur sacrifice aux divinités éthiopiennes ${ }^{128}$, on pense aussitôt que le thème apparaissait très probablement sous forme parodique dans l'épisode perdu du roman de Pétrone qui, selon le compte rendu de Servius $^{129}$, se déroulait à Marseille : là, lors d'une famine, un volontaire était nourri et soigné pendant une année entière, et ensuite traité en bouc émissaire. Chez Héliodore, naturellement, le roi reconnaît en Chariclée sa propre fille et le sacrifice est abandonné ; nous n'avons pas idée de la façon dont l'épisode se terminait chez Pétrone, mais l'intention désacralisante de l'auteur est quasiment certaine.

L'échange de lettres entre Encolpe et Circé ${ }^{130}$ a déjà été mentionnée par Heinze ${ }^{131}$, mais seulement en référence à la rhétorique et au style, sans qu'il ait été confronté avec les paires de lettres qui apparaissent dans les romans grecs - confrontation qui aurait démontré de toute évidence l'intention parodique de Pétrone. Nous reviendrons plus loin sur cet argument, étant donné que chez Pétrone la paire de lettres joue un rôle important au moment où il parodie plus largement la tentative que fait une rivale de l'héroïne pour séduire le héros masculin du roman - un thème courant dans le roman érotique grec. 
33 Nous achèverons cette partie de notre recherche en rappelant un motif ultérieur typiquement romanesque, dont la parodie apparait chez Pétrone : celui du double songe. Chez Chariton, Longus, Héliodore, Achille Tatius, et aussi chez Apulée ${ }^{132}$, nous rencontrons divers cas de deux, et une fois même de trois ${ }^{133}$ personnages dont les songes sont semblables ou en quelque façon visent le même résultat. Souvent il s'agit de songes prophétiques, dans lesquels les divinités donnent des avertissements ou prédisent l'avenir. Chez Longus apparaissent Eros et les Nymphes, chez Héliodore Apollon et Artémis, chez Achille Tatius Aphrodite, chez Apulée Isis et - probablement - aussi Osiris. Artémidore de Daldis nous informe ${ }^{134}$ qu'un rêve fait par plus d'une personne gagne en crédibilité, mais dans les romans le thème du double songe est utilisé plutôt comme instrument narratif efficace. L'importance de ce motif dans les romans d'amour à été amplement reconnu ${ }^{135}$.On peut dire la même chose de son emploi humoristique de la part de Pétrone ${ }^{136}$ dans la scène où Lichas et Tryphène se racontent leurs songes, par lesquels respectivement Neptune et Priape leur révèlent qu'ils trouveront Encolpe et Giton à bord du navire ${ }^{137}$. A part la joyeuse dégradation du motif inhérente à la nature des rapports personnels entre les personnages concernés, l'intention parodique de Pétrone paraît manifeste dans les paroles de Tryphène, quand elle écoute le récit d'un songe semblable au sien propre: «On diraitque nous avons dormi ensemble». Cette réplique montre que Tryphène et Lichas, qui pourtant n'ont rien de chaste, n'ont pas en réalité dormi ensemble ; au contraire, chez Héliodore, Théagène et Chariclée, purs et chastes pourtant, dorment cependant ensemble, et quand la jeune fille fait un cauchemar et hurle épouvantée, Théagène partage son agitation comme s'il avait fait le même songe ${ }^{138}$. Encore une fois, Pétrone a conféré un tour parodique à un motif typique amplement utilisé dans le roman antique et moderne. La Traumnovelle d'Arthur Schnitzler est entièrement centrée sur le thème du double songe : le songe d'Albertine et l'aventure de Fridolin se complètent réciproquement; et même si cette dernière est racontée de l'extérieur comme véritable, certains indices non équivoques en révèlent la nature onirique. Le mot de passe pour entrer dans la villa mystérieuse est 'Danemark' - le lieu dans lequel se déroule le songe d'Albertine; et à la fin Fridolin dira à sa femme : « aucun rêve n'est tout à fait un rêve ".

IV. Jusqu'ici nous avons rencontré des parodies de thèmes courants dans les romans grecs dans les parties des Satyrica qui empruntent extérieurement la structure ou les situations du roman d'amour. Nous ne nous attendons pas à trouver des parodies analogues dans les parties où Pétrone se tourne vers les autres sources et motifs d'inspiration qui convergent dans une œuvre si riche et variée que, comme nous l'avons déjà observé, on ne trouve pas de véritables parallèles dans la littérature antique. Il serait donc inutile de rechercher des parodies du roman d'amour, par exemple, dans le banquet de Trimalchion ou dans les discussions assez fréquentes sur la littérature. Mais que trouvons-nous dans les narrations insérées dans le récit principal, comme les histoires du garçon de Pergame ou de la matrone d'Éphèse, où a souvent été reconnue la trace fort évidente laissée par ce que l'on appelle les fabulae Milesiae?

S'agissant du premier de ces contes, on peut reconnaître une trace de parodie unique mais peut-être révélatrice en le confrontant avec une histoire racontée chez Achille Tatius et concernant non le couple principal du roman, mais le cousin de Clitophon, Clinias; elle met en scène un amour pédérastique comme celui que raconte Eumolpe. Clinias est si généreux qu'il fait cadeau à son aimé, Chariclès, d'un cheval qu'il avait acheté pour lui ${ }^{139}$; mais ce même cheval provoquera plus tard la mort de Chariclès ${ }^{140}$. Le 
thème tragique se trouve dégradé en parodie risible chez Pétrone, où le cheval n'existe pas, et où il n'est pas même réellement promis, mais seulement évoqué par Eumolpe dans le but d'obtenir les faveurs du garçon, dont il pourra encore jouir de façon répétée même une fois qu'il est devenu évident que le cheval ne se matérialisera jamais ${ }^{141}$.

Le conte de la matrone d'Éphèse est nettement plus complexe; non seulement il s'agit d'un thème diffusé dans nombre de littératures, orientales comme occidentales, mais nous pouvons aussi avoir la certitude que cette histoire particulière existait avant Pétrone, étant donné qu'elle apparaît dans une des fables de Phèdre ${ }^{142}$. Cela montre que ce conte d'aspect macabre avait reçu un traitement humoristique dans un type de littérature autre que les romans d'amour, dans lesquels, comme nous le verrons tout à l'heure, de nombreux éléments bien reconnaissables de l'histoire se trouvent pourtant encore utilisés avec un absolu sérieux. La confrontation avec Phèdre d'un côté et avec les romans d'amour de l'autre nous porte cependant à croire que Pétrone avait délibérément enrichi le conte par le biais de la déformation parodique de ces derniers.

Il n'est pas besoin de souligner que le thème de la mort apparente (Scheintod) est courant dans les romans grecs, et qu'il n'est pas rare qu'il y soit suivi de celui de l'ensevelissement de quelqu'un d'encore vivant. À une ou deux exceptions près ${ }^{143}$, c'est une femme qui est ensevelie vivante. Chez Chariton ${ }^{144}$, Callirhoé se trouve ensevelie vivante et elle est libérée par le pirate Théron. Chez Achille Tatius ${ }^{145}$ Leucippé est placée encore vivante dans un sarcophage. Chez Xénophon d'Éphèse ${ }^{146}$ Anthia se réveille dans un tombeau, et quand des voleurs y pénètrent elle leur demande de respecter sa chasteté - exactement le contraire de ce qui se passe dans le conte de Pétrone. Chez Héliodore la caverne souterraine dans laquelle est enfermée Chariclée est à plusieurs reprises définie comme une tombe (et Chariclée est décrite comme enterrée vivante ${ }^{147}$ ), mais elle a pour fonction de protéger sa chasteté1 ${ }^{148}$; à nouveau le contraire de ce qui arrive dans les Satyrica. Théagène la croit morte et dans son imagination la caverne devient la tombe dans laquelle lui et Chariclée reposeront, morts, ensemble ${ }^{149}$; cela correspond exactement à ce que croient les habitants d'Éphèse dans le récit de Pétrone : « la chaste épouse est morte sur le corps de son mari ", telles sont les paroles qui leur sont attribuées ${ }^{150}$. Inutile de dire que chez Pétrone la réalité est bien différente de cette façade honorable. Dans l'Histoire du roi Apollonios de Tyr ${ }^{151}$, la femme d'Apollonios est placée dans un cercueil jeté ensuite à la mer ; à Éphèse elle sera rappelée à la vie par un médecin.

L'histoire de la matrone d'Éphèse chez Pétrone parodie clairement le thème de la femme d'abord enterrée vive puis libérée ou rappelée à la vie ${ }^{152}$. Quand son mari meurt, elle n'est pas encore morte, mais désire mourir, et donc le suit dans la tombe; mais l'amour du soldat la ramènera à la vie : reuiuiscere, comme le dira sa servante ${ }^{153}$.

Curieusement, nous trouvons un renversement exact, encore qu'extrêmement sérieux, du motif macabre de la femme vivante demeurant dans la tombe avec son mari mort chez Xénophon d'Éphèse ${ }^{154}$. Ici le pêcheur Aigialeus conserve dans sa demeure le cadavre momifié de sa femme; dans ce cas ce n'est pas la femme vivante qui descend dans la tombe avec son mari défunt, mais le mari vivant qui tient sa femme défunte dans sa propre maison ${ }^{155}$. Apparemment Pétrone a tourné en dérision le motif de la fidélité amoureuse par-delà la mort.

40 V. Nous avons laissé pour la fin l'examen d'un ample épisode dans lequel, à ce qu'il me semble, l'usage parodique de la part de Pétrone de thèmes et motifs utilisés sérieusement dans le roman érotique grec est continuel et absolument assuré : l'aventure d'Encolpe avec Circé dans la partie finale de qui reste des Satyrica. 
41 La rivale féminine qui met à l'épreuve la fidélité du héros masculin de l'histoire apparaît dans plus d'un des romans grecs qui nous sont parvenus. Comme nous l'avons déjà observé, les héros grecs résistent jusqu'à la fin (à l'unique exception de Clitophon, dans la mesure où il cède au désir de Mélité une fois seulement et par compassion, avant de l'abandonner pour toujours ${ }^{156}$ ). En règle générale pourtant, dans les romans grecs, rien ne se passe, parce que les héros sont mal disposés à l'égard des avances que leur font les rivales de leurs amantes. Chez Pétrone au contraire Encolpe n'est que trop bien disposé, mais même dans ce cas il ne se passe rien, à cause de l'impuissance dont il est frappé. Chez Pétrone l'infirmité est réelle, mais le thème de l'impuissance apparaît aussi dans les romans grecs, comme une fiction dans le but de repousser les appétits des rivales: comme une excuse inventée par l'entremetteuse pour justifier son propre insuccès chez Héliodore ${ }^{157}$, ou par le héros lui-même, comme le fait Clitophon à l'adresse de Mélité chez Achille Tatius ${ }^{158}$, avec des paroles assez voisines de certaines répliques d'Encolpe ${ }^{159}$. Incidemment, si Pétrone s'est amusé à frapper réellement son Encolpe de la maladie habituellement alléguée comme excuse par les héros des romans d'amour lorsque leur vertu était mise à l'épreuve par une rivale de leur amoureuse, il n'y a pas de raison de croire qu'Encolpe souffre de crises d'impuissance dans d'autres parties du roman que l'épisode de Circé, comme le pensent certains savants. Assurément Encolpe ne se montre absolument pas impuissant dans les parties précédentes du récit ${ }^{160}$, et il apparaît non seulement affligé, mais aussi surpris de sa faillite avec Circé : comme il le dit lui-même, il avait l'habitude d'être un Achille dans les jeux amoureux ${ }^{161}$. Sachant que Clitophon, à la fin, satisfait la passion de Mélité, nous ne pouvons pas non plus être sûrs qu'Encolpe aussi, à la fin, ne réussirait pas à aimer Circé ; de fait il paraît encore amoureux d'elle peu après ${ }^{162}$ avoir définitivement récupéré sa propre virilité ${ }^{163}$; mais l'état de la tradition ne nous offre aucun indice.

Le thème de l'impuissance n'est que l'un des nombreux fils composant la trame du thème, courant dans les romans d'amour, relatif à la rivale féminine de l'héroïne. Chez Héliodore, Cybèle, l'entremetteuse, choisit une approche indirecte pour persuader Théagène ${ }^{164}$ et ensuite pour vaincre sa résistance ${ }^{165}$. La situation apparaît nettement inversée chez Pétrone, tout comme l'attitude d'Encolpe va à l'encontre de celle des héros grecs : Chrysis, une des entremetteuses de Circé, est directe à en être brutale ; elle ne s'attend à aucune résistance de la part d'Encolpe, à l'exception peut-être d'une demande d'argent ${ }^{166}$. Mais, comme nous l'avons déjà vu ${ }^{167}$, Cybèle fait référence au même épisode homérique - la scène d'amour entre Zeus et Héra sur le mont Ida - qui joue un rôle important dans l'épisode de Circé chez Pétrone. Elle finira aussi par subir un sort semblable à celui de l'autre entremetteuse de Pétrone : Cybèle est en fait rudement chassée ${ }^{168}$, tout comme la sorcière Proselenos dans les Satyrica, tandis que Chrysis est fouettée ${ }^{169}$.

La femme amoureuse de Théagène n'est rien moins que l'épouse du satrape perse d'Égypte, mais même Circé est une dame respectable: une matrona ${ }^{170}$; et dans Achille Tatius Mélité est une veuve riche et respectée - ou du moins on la croit telle, jusqu'à la réapparition de son mari considéré comme mort. Néanmoins elle est tout à fait capable de demander tout particulièrement à Leucippé, qu'elle prend pour une sorcière éprouvée, de lui préparer un philtre dans le but d'obtenir l'amour de Clitophon. En accord avec la situation qui prévaut dans les romans grecs, on recourt à la magie pour vaincre la résistance du héros masculin ; chez Pétrone, naturellement, on devra l'employer pour tenter de guérir la véritable impuissance physique d'un Encolpe qui sur le plan psychologique ne résiste nullement ${ }^{171}$. 
44 À la différence d'Encolpe, Clitophon - nous l'avons vu - feint l'impuissance afin de faire perdre son temps à Mélité, qui de son côté use de mots quasi identiques à ceux que prononce Encolpe à propos de Circé. Elle dit en effet : «bien que je sois détestée, j'aime celui qui me déteste; bien que je souffre, je suis attirée par celui qui me fait souffrir; l'humiliation ne m'empêche pas d'aimer " "172, "Même si j'ai des motifs de me fâcher, je brûle de passion; bien qu'humiliée, j'aime ; fais la paix avec moi, aie pitié de moi $»^{173}$. Encolpe de son côté dit : «Les humiliations ne me rebutent pas ; j'ai été battu, mais je l'ai oublié ; j'ai été chassé, mais je le prends comme un jeu; puissé-je seulement rentrer en grâce auprès d'elle! $»^{174}$. Nous assistons ici à un échange des rôles sexuels, qui se répète régulièrement dans l'épisode de Circé, comme je crois l'avoir montré ailleurs ${ }^{175}$.

Quand Thersandre, le mari de Mélité que l'on avait faussement cru mort, réapparaît à l'improviste, il flanque à Clitophon une bruyante correction ${ }^{176}$. Le parallèle parodique chez Pétrone est la raclée que donnent à Encolpe les esclaves de Circé177. Chez Achille Tatius Clitophon est battu parce que Thersandre croit - à tort - qu'il a eu des rapports sexuels avec Mélité. Il n'aurait pas été battu, si Thersandre avait su la vérité. Ni chez Achille Tatius ni chez Pétrone il n'y a eu de rapport sexuel quelconque, mais Thersandre bat Clitophon parce qu'il ne le sait pas, tandis que Circé fait battre Encolpe parce qu'elle ne le sait que trop bien. Thersandre se sent offensé parce qu'il croit que Clitophon a eu des rapports avec sa femme; Circé est offensée parce qu'elle sait qu'Encolpe n'a pas eu de rapports avec elle. De même chez Xénophon d'Éphèse Habrocomès est battu et torturé parce qu'on croit - à tort - qu'il a eu des rapports avec Manto ${ }^{178}$, tandis qu'en réalité il a repoussé ses avances. Dans aucun des cas considérés il n'y a eu de rapport sexuel quelconque; dans les romans grecs cependant, apparaissent des menaces de punition lorsque le héros masculin ne satisfait pas le désir de la rivale de son amante, soit pour la même raison qui fait qu'Encolpe est puni chez Pétrone. Cela arrive chez Héliodore, quand Cybèle fait allusion à la vengeance d'Arsacé, dans le cas où Théagène refuserait de satisfaire sa passion, et chez Xénophon d'Éphèse des menaces analogues sont contenues dans la lettre que Manto écrit à Habrocomès ${ }^{179}$.

La manière dont Pétrone traite ce motif se comprend mieux à travers la confrontation avec la scène finale ${ }^{180}$ de Lucius ou l'Âne attribuée à Lucien. Après avoir repris forme humaine, Lucius va rendre visite à la dame qui avait été son amante quand il était un âne. L'épisode correspondant dans les Métamorphoses d'Apulée ${ }^{181}$ nous apprend qu'il s'agit d'une respectable matrona, comme Circé. Naturellement, sous sa forme humaine, Lucius est incapable de la satisfaire comme il le faisait en tant qu'âne. Ainsi, le motif de la déception de la dame n'est pas très différent de celui de la déception analogue de Circé dans les Satyrica. Comme Circé, elle aussi appelle ses esclaves et fait chasser Lucius. Celuici reçoit des crachats, tout comme Encolpe ${ }^{182}$ - tout correspond, sinon qu'il n'est pas fait mention expresse de la rossée. L'influence de la Milésienne dans cet épisode est démontré par un fragment de la traduction latine de Sisenna, qui trouve un parallèle précis tant chez le Pseudo-Lucien que chez Apulée ${ }^{183}$. Il n'est pas impossible, donc, que Pétrone ait donné à un motif courant dans la littérature narrative antique un tour comique semblable à celui qu'il avait déjà reçu dans la Milésienne; mais les nombreuses parodies, dans tout l'épisode, de thèmes utilisés sérieusement dans les romans d'amour font qu'on ne peut que se dire que, en plus du modèle à suivre de la Milésienne, Pétrone, dans ce cas aussi, avait à l'esprit également ces derniers, comme 'anti-modèle' à parodier.

On en trouve une confirmation ultérieure dans la façon dont est traité chez Pétrone le thème de l'échange épistolaire entre les amoureux ${ }^{184}$. Un échange analogue figure chez 
Achille Tatius, non entre la rivale féminine et le héros de l'histoire, mais entre Leucippé et Clitophon, c'est-à-dire entre le héros et l'héroïne ${ }^{185}$. Comme chez Pétrone, la première à écrire est la dame, et ici aussi elle a des motifs de lamentation, quoique différents de ceux de Circé. Clitophon lui répond en lui assurant qu'il n'a pas eu de rapports avec Mélité, tout comme Encolpe n'en a pas eu avec Circé ; mais naturellement, ce qui constitue un mérite pour Clitophon est en revanche la faute la plus grave dont se soit souillé Encolpe. Chez Chariton une lettre de Chéréas à Callirhoé ne reçoit pas de réponse, parce qu'elle est interceptée par Dionysios. Dans cette lettre Chéréas admet ses torts à l'égard de Callirhoé, tout comme Encolpe à l'égard de Circé, même si, inutile de le dire, il s'agit de torts d'une nature bien différente.

Mais le parallèle le plus étroit est naturellement l'échange épistolaire entre Manto, la rivale d'Anthia, et Habrocomès, le héros de l'histoire, chez Xénophon d'Éphèse ${ }^{186}$. Comme Circé, Manto est la première à écrire ; elle propose sérieusement à Habrocomès de tuer sa rivale Anthia. La façon dont Pétrone transforme ce motif cruel fait sourire le lecteur: Circé serait plus que prête à accepter Giton comme rival ${ }^{187}$; et à l'inverse c'est Encolpe, intervertissant encore un fois les rôles sexuels, qui se déclare prêt à sacrifier Giton ${ }^{188}$ - sacrifice ironiquement refusé par Circé189. Chez Xénophon d'Éphèse Habracomès répond en refusant les avances de Manto avec de nobles paroles : « Dame, fais comme bon te semble et traite mon corps comme celui d'un esclave ; si tu veux me tuer, je suis prêt, si tu veux me torturer, agis à ta convenance; mais je ne viendrai pas dans ton lit, et si tu me l'ordonnais, je n'obéirais pas. $»^{190}$ Encolpe use de mots quasi identiques dans sa réponse à Circé : «si tu veux me tuer, j'arrive avec ma propre épée; s'il te suffit de me battre, j'accours nu vers ma dame ${ }^{191}$; sinon que, naturellement, tout au contraire d'Habracomès, il serait trop heureux d'avoir une seconde occasion d'entrer dans le lit de Circé.

De toute évidence Pétrone a repris un motif romanesque: l'amour d'une rivale de l'héroïne pour le héros de l'histoire - amour destiné à ne pas se réaliser du fait du refus obstiné du héros. Il fait la parodie de ce thème en nous présentant un Encolpe plus que disposé à satisfaire la dame, dont le désir ne se réalise pas non du fait de la chasteté du héros, mais du fait de son impuissance - qui est réelle, qui n'est pas une simple excuse, comme dans les romans grecs. Pour cette raison l'échange épistolaire est placé après la première rencontre amoureuse manquée.

Si nous nous souvenons de l'épisode de Circé chez Pétrone et des parallèles que nous venons de mettre en évidence dans les romans grecs, nous serons en situation, je pense, de juger si le tour comique et désacralisant, conféré dans les Satyrica de façon continue et cohérente à des thèmes et motifs qui, dans les romans grecs d'amour, sont utilisés sérieusement, peut être attribué à un simple hasard; et, s'il existe un rapport, nous jugerons si c'est l'usage sérieux ou l'usage parodique qui a précédé l'autre dans le temps. Dans aucun des deux cas, me semble-t-il, il ne peut y avoir raisonnablement de doute. 


\section{BIBLIOGRAPHIE}

J. Adamietz, « Zum literarischen Charakter von Petrons Satyrica”, RhM 130, 1978, p. 329-346.

A. Aragosti, Petronio Arbitro. Satyricon. Introd., trad. e note. Testo latino a fronte, Milano 1995.

G. Arrigoni, “Amore sotto il mantello e iniziazione nuziale”, QUCC 15, 1983, p. 7-56.

R. Astbury, "Petronius, P.Oxy. 3010, and Menippean Satire", CPh 72, 1977, p. 22-31.

B. Baldwin, "Ira Priapi”, CPh 68, 1973, p. 294-296.

M. Brioso “Sánchez, Heliodoro VI 5-11 y la crisis del ‘amigo' en la novela”, Habis 18-19, 1987-1988, p. 101-107.

C. Corbato, "Da Menandro a Caritone. Studi sulla genesi del romanzo greco e i suoi rapporti con la commedia nuova", Quaderni Triestini sul Teatro Antico 1, 1968, p. 5-44.

F.M. Fröhlke, Patron. Struktur und Wirklichkeit. Bausteine einer Poetik des antiken Romanes, FrankfurtBern 1977.

L. Galli, "Meeting again. Some Observations on Petronius Satyricon 100 and the Greek Novels", in : H. Hofmann - M. Zimmermann (eds.), Groningen Colloquia on the Novel 7, Groningen 1996, p. 33-45

P. Habermehl, Petronius, Satyrica 79-141. Ein philologisch-literarischer Kommentar. Band I: Sat. 79-110, Berlin-New York 2006

T. Hägg, The Novel in Antiquity, Oxford 1983.

R. Heinze, "Petron und der griechische Roman", Hermes 34, 1899, p. 494-519.

R. Heinze, Vom Geist des Römertums, Stuttgart $1960^{3}$.

R. Helm, Der antike Roman, Göttingen $1956^{2}$.

N. Holzberg, Der antike Roman. Eine Einführung, München-Zürich 1986.

K. Kerényi, Die griechisch-orientalische Romanliteratur in religionsgeschichtlicherBeleuchtung, Tübingen 1927.

E. Klebs, “Zur Composition von Petrons Satirae”, Philologus 47, 1889, p. 623-635.

P. Kragelund, "Epicurus, Priapus and the Dreams in Petronius", CQ (NS) 36, 1989, p. 436-450.

H. Kuch, “Gattungstheoretische Überlegungen zum antiken Roman”, Philologus 129, 1985, p. 3-19.

F. Létoublon, "La lettre dans le roman grec ou les liaisons dangereuses", in : S. Panayotakis - M. Zimmermann - W. Keulen, The Ancient Novel and beyond, Leiden-Boston 2003, p. 271-288.

P. Liviabella, Strategie macro-retoriche della comunicazione nel romanzo di Eliodoro, in corso di stampa.

S. MacAlister, Dreams and Suicides. The Greek Novel from Antiquity to the Bizantine Empire, LondonNew York 1996.

N. Marini, “Drama: possible denominazione per il romanzo d'amore”, SIFC 84, 1991, 232-242.

C.W. Mendell, Petronius and the Greek Romance", CPh 12, 1917, p. 158-172. 
R. Merkelbach, Roman und Mysterium in der Antike, München-Berlin 1962.

J. Morgan, Kleitophon and Encolpius: Achilles Tatius as Hidden Author",in: The Greek and Roman Novel. Parallel Readings, Ancient Narrative Suppl. 8, Groningen 2007, p. 105-120.

M. Pacchieni, Nota petroniana. L'episodio di Circe e Polieno (capp. 126-131; 134)", BStudLat 6, 1976, p. 79-90.

C. Panayotakis, Theatrum Arbitri. Theatrical Elements in the Satyrica of Petronius, Leiden 1995.

A.D. Papanikolaou, Chariton-Studien. Untersuchungen zur Sprache und Chronologie der griechischen Romane, Göttingen 1973.

P.J. Parsons, A Greek Satyricon?", BICS 18, 1971, p. 53-68.

P.J. Parsons, Narrative about Iolaus (POxy 3010), The Oxyrhynchus Papyri 42”, 1974, p. 34-41.

B.E. Perry, Petronius and the Comic Romance", CPh 20, 1925, p. 31-49.

B.E. Perry, On Apuleius' Hermagoras", AJPh 48, 1927, p. 263-266.

B.E. Perry, The Ancient Romances. A Literary-Historical Account of their Origins, Berkeley-Los Angeles 1967.

M. Plastira-Valkanou, Dreams in Xenophon Ephesius", SO 76, 2001, p. 137-149.

E. Rohde, Der griechische Roman und seine Vorläufer, Leipzig $1914^{3}$.

C. Ruiz-Montero, The Rise of the Greek Novel", in : G. Schmeling (ed.), The Novel in the Ancient World, Boston-Leiden $2003^{2}$, p. 29-85.

G.N. Sandy, "Satire in the Satyricon”, AJPh 90, 1969, p. 293-303.

G.N. Sandy, "Notes on Lollianus" Phoenicica, AJPh 100, 1979, p. 367-376.

A. Scobie, Aspects of the Ancient Romance and its Heritage. Essays on Apuleius, Petronius, and the Greek Romances, Meisenheim am Glan, 1969.

A. Setaioli, “Cinque poesie petroniane (Sat. 82.5, 83.10, 108.14, 126.18, 132.15)", Prometheus 24, 1998, p. 217-242.

A. Setaioli, "La poesia in Petr. Sat. 127.9", Prometheus 25, 1999, p. 247-258.

A. Setaioli, “La poesia in Petr. Sat. 79.8”, Prometheus 27, 2001, 136-144.

A. Setaioli, “La poesia in Petr. Sat. 80.9”, Prometheus 27, 2001, 57-72 (2001a).

A. Setaioli, "Le due poesie in sotadei di Petronio (Sat. 23.3; 132.8)", Cuadernos de Filología Clásica. Estudios Latinos 23, 1, 2003, p. 89-106.

S.A. Stephens - J.J. Winkler, Ancient Greek Novels. The Fragments. Introduction, Text, Translation, and Commentary, Princeton, 1995.

Chr. Stöcker, Humor bei Petron, Diss. Erlangen-Nürnberg, 1969.

G. Thiele, “Zum griechischen Roman”, in: Aus der Anomia. Archäologische Beiträge Carl Robert dargebracht, Berlin 1890, p. 124-133.

F. Wehrli, “ 'Meeting' Einheit und Vorgeschichte der griechisch-römischen Romanliteratur”, MH

22, 1965, p. 133-154. 


\section{NOTES}

1. On peut se reporter à Scobie 1969,3, et Adamietz 1987, 330, comme exemples de chercheurs mettant l'accent sur le caractère unique de l'œuvre de Pétrone, même si tous deux finissent par donner de l'importance à l'influence de la satire - surtout la satire ménippée.

2. À voir maintenant dans l'édition Stephens -Winkler 1995, 314-357.

3. Ach. T. $3,15,6$.

4. Cf. par ex. Sandy 1979. Nous pouvons ajouter les flatulences (Lollianus B.1 verso, 12; cf. Petr. 117,12)

5. Parsons 1971; Parsons 1974. Désormais éd. Stephens-Winkler 1995, 358-374. Pour le fragment de Iolaus et Pétrone, voir Setaioli 2003, avec la bibliographie citée et discutée.

6. Petr. 23,3. Autres vers sotadiques dans 132.8 .

7. Stephens-Winkler 1995, 400-408.

8. Cette position a été soutenue par Astbury 1977

9. Sud. IV, p. 724, 25-26 Adler.

10. Ov. trist. 2,415-417.

11. Cette œuvre est citée par Priscian II 85 Keil, etc., et Fulg. exp. serm. ant. 3. Voir Perry 1927.

12. Pour les différents types de fiction cf. par ex. Helm $1956^{2}$; Kuch 1985; maintenant RuizMontero $2003^{2}$.

13. Rohde $1914^{3}$.

14. Macr. Somn. Scip. 1,2,8 correspond exactement à Julien, ep. 48 Weis, 301B. Même si Macrobe met sur le même plan les histoires de Pétrone et d'Apulée et les pièces de Ménandre, tandis que Julien fait référence à la forme historiographique, tant argumenta (chez Macrobe) que hypotheseis (chez Julien) peut impliquer une allusion au théatre. Sur ces passages cf. Kuch 1985, 9-10.

15. Par ex. Mendell 1917, 160; Wehrli 1965, 134; 140; 147; 152.

16. Heinze 1899 (= Heinze $\left.1960^{3}, 417-439\right)$. Les citations font référence à l'article de 1899.

17. Heinze 1899, 518 n. 3.

18. Par ex. Stöcker 1969, 3 sq.; Hägg 1983, 171 sq.; Holzberg 1986, 73 sq. Dernièrement Habermehl 2006, 258

19. Cf. Papanikolaou 1973, 9-19; 163.

20. Cf. Stephens-Winkler 1995, 23-71.

21. Ainsi, par ex., Pers. 1,134 post prandia Callirhoen do, qui pourrait être une référence au roman de Chariton. Thiele 1890 pensait qu'on pouvait voir une allusion au roman dans Cic. inv. 1,27 (cf. Rhet. Her. 1.12-13). Cela ne semble pas fondé ; voir cependant Perry 1967, 75; 144-146.

22. Cf. le schéma ap. Stöcker 1969, 5 n. 1 (tentatives de suicide; tempête en mer et naufrage ; procès; rivaux amoureux; mort apparente; monologues; ekphrasis; sentences; assemblées populaires; double songe). Galli 1996 ajoute le thème de la seconde rencontre entres personnages.

23. Cf. déjà Heinze 1899, 503.

24. Cf. Heinze 1899, 504. Quand Wehrli 1965, 147, parle de 'polarité' en référence à l'usage sérieux et à l'usage bouffon des mêmes thèmes, cela équivaut à éviter le problème de l'antériorité de l'un ou de l'autre.

25. Cf. Mendell 1917, 165.

26. Cf. par ex. Apul. met. 2,19-31

27. Cf. par ex. Sandy 1969, et ci-dessus, note 1 .

28. Perry 1925, 37, pense que la parodie de l'épopée chez Pétrone montre qu'il n'avait pas l'intention de faire une parodie continue du roman d'amour. Pour Adamietz 1987, 342, le roman érotique grec n'est que l'un des nombreux genres littéraires parodiés par Pétrone. Même si cela 
est assurément exact, ce qui lui échappe, c'est que la parodie des genres littéraires élevés ne peut être isolée de celle qui vise les romans d'amour.

29. Pour Mendell 1917, 172, et Perry 1925, 37, la parodie du roman d'amour dans les Satyrica est purement épisodique. Stöcker 1969, 4, observe plus judicieusement qu'elle ne se rencontre pas toujours et partout ; Adamietz 1987, 333, remarque lui aussi que les Satyrica n'accordent pas à la trame amoureuse la même prééminence que dans les romans grecs.

30. Petr. 81-90.

31. Hist. Apoll. reg. Tyr. 18, p. 13, 19-21 Schmeling (Redactio A).

32. Cf. Setaioli 2003, 98-100, pour l'usage parodique de l'épisode de Didon dans 132,8-11 et l'histoire de la veuve d'Éphèse $(111,12 ; 112,2)$.

33. Petr. 118,5 ; cf. 68,5 .

34. Petr. 127,9.

35. Heliod. 7,21,2. Référence à Il. 14,344-345.

36. Cf. Setaioli 1999.

37. Petr. $127,9,7$ secreto favit amori.

38. Petr. 126,18. Cf. Setaioli 1998, 232-237.

39. Petr. 132,13

40. Odyss. 20,18 sq.

41. Soph. Oed. t. 1270 sq.

42. Cf. Charit. 6,1,9 (le roi de Perse prend pitié de ses yeux parce que bientôt ils ne verront plus Callirhoé); hist. Apoll. reg. Tyr. 38 (Apollonios tance ses propres yeux pour n'avoir pas pleuré en lisant l'inscription qui atteste faussement la mort de Tarsia). Dans un passage narratif conservé dans un fragment de papyrus (PSI 981), Calligoné maudit ses propres yeux pour avoir vu un certain Erasinus (cf. Stephens-Winkler 1995, 272, lines 18-20).

43. Heinze 1899, 495-496

44. Par ex. Wehrli 1965, 136-137. Si chez Antonius Diogenes Demochares n'est pas en réalité le fils plutôt que l'amant de Dinias (comme l'a suggéré Wehrli 1965, 137), il ne joue assurément pas de rôle érotique identifiable dans le sommaire présenté par Photius. Dinias tombe amoureux d'une femme, Derkyllis.

45. Cf. par ex. Adamietz 1987, 332; et déjà Heinze 1899, 497 n. 3.

46. Ach. T. 1,2,1 (Zeus et Europe); Long. soph. prooem. (Daphnis et Chloé). Le thème corrélé du pouvoir exercé par l'amour sur les dieux aussi apparaît également ailleurs (Ach. T. 1,5,7: Apollon et Daphné; Charit. 3,3,6: Thétis and Pélée; cf. Petr. 83,4 ergo amor etiam deos tangit). Cf. Aragosti 1995, 338-339 nn. 244-245.

47. Petr. 83,3-5: Jupiter et Ganymède; Hylas, l'amant d'Hercule, repoussant une nymphe - refus qui n'est pas attesté ailleurs (cf. Habermehl 2006, 73); Apollon and Hyacinthe.

48. Petr. 79,8 .

49. Setaioli 2001, avec une discussion détaillée des parallèles textuels; en particulier Ach. T. 2,8,2; 2,37,9-10. L'interprétation que fait Habermehl 2006, 9-10, du dernier vers du poème n'est pas, à mon avis, convaincante.

50. Le thème apparaît aussi dans un autre roman d'amour: Xenoph. Eph. 1,9,6: les âmes des amants communiquent à travers le baiser; voir aussi Ach. T. 4,8,2.

51. Petr. 132,1.

52. Petr. respectivement 91,6 et 138,8 .

53. Petr. $140,11$.

54. Par ex. Petr. 91,7.

55. L'Arioste, Orl. fur. 27,104-109.

56. Charit. 5,8-10.

57. Petr. 81,3 . 
58. Petr. 80,6. La relation parodique avec la scène de Chariton semble avoir échappé à Habermehl 2006, 20-23, comme à tous les commentateurs de Pétrone.

59. Petr. 133,2 sibi ab Ascylto nullam vim factam

60. Heinze 1899, 501.

61. On peut se demander si le fait que Giton et Encolpe ne restent physiquement séparés que pendant un petit nombre de chapitres ne doit pas être interprété lui aussi comme une parodie ; cependant, même physiquement proches, les deux 'héros' de Pétrone sont bien plus éloignés l'un de l'autre que ne le sont les amants séparés des romans grecs.

62. Cf., par exemple, Wehrli 1965, 139. Selon certains savants, le Cnémon d'Héliodore rompt le lien d'amitié quand il quitte Chariclée : Brioso Sánchez 1987-1988; Liviabella (sous presse).

63. Par ex. Heinze 1899, 501.

64. Klebs 1889.

65. Cf. Baldwin 1973.

66. Par ex. Adamietz 1987, 334.

67. Petr. 115,4 .

68. Heliod. 5,24,5.

69. Heliod. 5.24,2.

70. Ach. T. 3,3-4.

71. Petr. 114,7 .

72. Petr. 102,2-5.

73. Heliod. 5,51.

74. Petr. 79,4. Habermehl 2006, 3, ne fait pas référence à Héliodore.

75. Long. soph. 3,6,1-2.

76. Petr. 109,7. Cf. Habermehl 2006, 468.

77. Petr. 115,11-20.

78. Long. soph. 1,31 .

79. Ach. T. 1,15.

80. Petr. 131,8 .

81. Ach. T. 1,4,2-3.

82. Ach. T. 5,13.1. Cf. la description d'Anthia chez Xenophon (1,2,5-6).

83. Circé est comparée à la Diane de Praxitèle chez Petr. 126,16; Leucippé à un portrait d'Europe chez Ach. T. 1,4,3 (les manuscrits ont Selēnēn. Cf. Morgan 2007, 113 n. 31).

84. Petr. 126,18 .

85. Ach. T. 1,11,2.

86. Stöcker 1969, 9; Adamietz 1987, 334-335.

87. Petr. 114,8-12.

88. Cf. par ex. Wehrli 1965, 142.

89. L'un des parallèles les plus proches de la scène pétronienne se rencontre chez Heliod. 5,24,3: Chariclée, à bord du navire, veut mourir entre les bras de Théagène du même coup d'épée; mais le désir de mourir ensemble est un des motifs les plus communs de tous les romans.

90. Heliod. 10,19,2. Bien d'autres passages pourraient être cités de la même façon.

91. Petr. 94,8. Cf. Habermehl 2006, 258.

92. Charit. 5,10,6. D'autres pendaisons n'entraînant pas la mort figurent chez Apul. met. 1.16 et dans le sommaire des Babyloniaka de Jamblique transmis par Photius (§ 18).

93. Charit. 5,10,6 ff. De semblables lamentations apparaissent ailleurs aussi chez Chariton. Cf. Habermehl 2006, 34 .

94. Petr. $81,2 \mathrm{ff}$.

95. Apul. met. 8,14. Un autre suicide véritable (et double) - mais pas dans un contexte érotique se rencontre chez Apul. met. 9.38.

96. Petr. 94,13. 
97. Petr. 94,9-11.

98. Petr. 94,10. Habermehl 2006, 267-268, observe à juste titre que Giton joue un rôle dans tout l'épisode.

99. Petr. 9,5; 80,1.

100. Petr. $94,13$.

101. Petr. 94.15. En 95,1 la situation toute entière est assimilée à une représentation théâtrale : dum haec fabula inter amantes luditur. Cf. Habermehl 2006, 267.

102. Ach. T. 3,20-21. Cf. en dernier lieu Habermehl 2006, 266.

103. La tournure parodique de la situation chez Pétrone est reconnue par Wehrli 1965, 143 n. 23; Adamietz 1987, 336 n. 25.

104. Petr. 108,10-11.

105. Heliod. 1,4,1. Aucun parallèle romanesque n'est mentionné par Habermehl 2006, 449.

106. Panayotakis 1995.

107. Cf. par ex. Corbato 1968.

108. Macr. somn. Scip. $1,2,8$.

109. Cf. Marini 1991, qui montre en outre comment le terme de drama a souvent été utilisé pour désigner le roman d'amour.

110. Cf. Petr. 95,1 (cité ci-dessus, note 101); 92,13; et surtout le poème de 80,9, au sujet duquel on consultera Setaioli 2001a; Habermehl 2006, 24-31, avec la bibliographie citée et discutée.

111. Petr. 94,15 , même si ici la "théâtralité " au sens propre du terme est elle aussi au premier plan. Le mimicus risus de 19,1 montre probablement que Quartilla suit un scénario préparé d'avance dans l'exécution de sa propre cérémonie licencieuse.

112. Petr. 106,1.

113. Petr. 117,4 .

114. Petr. 140,6 .

115. Petr. 108,11 .

116. Cf. par ex. Heliod. 2,1,21; 10,12,2; Ach. T. $5,21,3 ; 7,11,1 ; 8,8,14$; etc.

117. Odyss. 6.154 sq. (paroles d'Ulysse à Nausicaa).

118. Charit. 1,1,16; 8,5,8; Xenoph. Eph. 1,2,7; 1,7,3; cf. 2,2,4; hist. Apoll. reg. Tyr. 31.

119. Apul. met. 11.16 et 22 .

120. Petr. 94,1-3. Pas de référence à des parallèles romanesques chez Habermehl 2006, 252.

121. Xenoph. Eph. 1,2,7.

122. Hist. Apoll. reg. Tyr. 31

123. Luc. $11,27$.

124. Cf. Setaioli 1999, 256 n. 65, pour Archiloque; et, pour une présentation d'ensemble, Arrigoni 1983.

125. Long. soph. $3,24,2$.

126. Petr. 11,2-3.

127. Petr. 114,9-12.

128. Heliod. 9,25,4.

129. Serv. Ad Aen. 3.57.

130. Petr. $129-130$.

131. Heinze 1899, 516. L'échange parallèle de lettres d'amour chez Pétrone et dans les romans grecs n'a fait l'objet que d'une rapide remarque de la part de Pacchieni 1976, 79-80; 90.

132. Charit. $1,12,5$ avec $1,12,10$ et $2,1,2$; Long. soph. $1,7,1$ et 2,$1 ; 2,10,1 ; 4,34,1$ et $4,35,5$; Heliod. $8,11,1-4 ; 9,25,1$ et $10,3,1$; cf. 2,16,1-2; 3,11,5; Ach. T. $1,3,4$ et 2,11,1; 4,1,3-8; Apul. 11,6; 11,22; $11,27$.

133. Charit. $1,12,5$ avec $1,12,10$ et $2,1,2$ (Théron, Léonas, Dionysios). Dans ce cas les songes sont différents, mais leur effet est le même.

134. Artemid. onirocr. 1.2, p. 10, 21-23 Pack. 
135. Cf. par ex. Kerényi 1927, 166; Merkelbach 1962, 132; plus récemment Galli 1996, 42; Habermehl 2006, 384-387. Sur les rêves dans le roman grec voir en dernier lieu MacAlister 1996; Plastira-Valkanou 2001.

136. Cf. Adamietz 1987, 336 n. 25; Kragelund 1989, 437-439, ainsi que sa polémique avec Fröhlke 1977, 40-42, qui considère que l'intégration du thème du double songe dans le contexte pétronien n'est pas une réussite.

137. Petr. 104,1-2.

138. Heliod. 2,16,2.

139. Ach. T. $1,7,1$.

140. Ach. T. 1,12 .

141. Petr. $86-87$.

142. Phaedr. app. 13.

143. Chez Apul. met. 10,5-12 un garçon est enseveli vivant,mais l'élément érotique est absent; selon le témoignage de Photius ( $\$$ ), dans les Babyloniaka de Jamblique Rhodanes et Sinonis dînent et dorment à l'intérieur d'un tombeau ; cependant ils ne sont pas ensevelis vivants.

144. Charit. 1,8-9.

145. Ach. T. 3,22 .

146. Xenoph. Eph. 3,8,1-5.

147. Heliod. 2,4,4; 5,2,8; Chariclée est décrite comme enterrée vivante dans 1,29,4.

148. Heliod. 1,28-29.

149. Heliod. $2,4,4$.

150. Petr. 112,3.

151. Hist. Apoll. reg. Tyr. 25-26.

152. Merkelbach 1962 croyait que ce thème symbolisait des doctrines mystiques de résurrection. Tout comme son interprétation générale du roman grec, cela est difficilement crédible; cf. par ex. Wehrli 1965, 142.

153. Petr. 111,12. l'ancilla dira aussi vivas. Cf. vivere (\$13).

154. Xenoph. Eph. 5,1,9-11.

155. Il s'agit peut-être d'une réminiscence d'Eurip. Alc. 348-352.

156. Ach. T. 5,27.

157. Heliod. 7,19,9. Cybèle, l'entremetteuse, dit à Arsacé que Théagène ne peut pas la satisfaire, parce qu'il est souffrant.

158. Ach. T. 5,21 .

159. Ach. T. 5,21,3: «Je ne sais pas ce qui m'arrive ; une maladie s'est emparée de moi »; cf. Petr. 128,2: « un sortilège s'est abattu sur moi »; 129,1: « je ne sais plus si je suis un homme, je ne me reconnais plus tel ».

160. Petr. 11,1; 79, 8. L'impuissance momentanéd d'Encolpe dans la 'cérémonie' de Quartilla est due ou à l'épuisement $(20,2)$ ou au dégoût $(23,5)$.

161. Petr. $129,1$.

162. Petr. 138,6-8.

163. Petr. $140,12-13$.

164. Heliod. 7,19.

165. Heliod. 7,20.

166. Petr. 126,1-4.

167. Cf. ci-dessus, note 35 .

168. Heliod. 7,22,2.

169. Petr. 132,5.

170. Petr. 126,$11 ; 132,2$.

171. Petr. 131 sq.

172. Ach. T. 5,25,2. 
173. Ach. T. $5,26,1$.

174. Petr. 138,8. Encolpe a la même attitude aussi à l'égard de Giton : 91,6.

175. Setaioli $1999,254-257$

176. Ach. T. 5,23 .

177. Petr. $132,2-4 ; 138,7$.

178. Xenoph. Eph. 2,6.

179. Heliod. 7,20,4-5; Xenoph. Eph. 2,5,2.

180. Ps. Lucian. Luc. sive asin. 56.

181. Apul. met. 10,19-22.

182. Lucius se fait cracher dessus par la dame, Encolpe par les esclaves (Petr. 132,3).

183. Sisenna Miles. libri fr. 9 Peter $=10$ Buecheler; cf. Ps. Lucian. Luc. sive asin. 51 et Apul. met. 10.22 .

184. Petr. 129-130. Je ne vois pas comment Adamietz 1987, 336 n. 25, peut affirmer que le motif de l'échange de lettres chez Pétrone n'a aucun rapport avec les cas analogues dans les romans grecs. Létoublon 2003 ne fait aucune référence à Pétrone.

185. Ach. T. 5,18,3-6 and 5,20,5.

186. Xenoph. Eph. 2,5, 1-2 et 4. Dans le roman de Xénophon se trouve encore un autre épisode, moins développé, qui met en scène une rivale de l'hérö̈ne: Kyno $(3,12)$.

187. Petr. 127,2 .

188. Petr. $127,3$.

189. Petr. 127.4.

190. Xenoph. Eph. 2,5,4.

191. Petr. $130,3$.

\section{RÉSUMÉS}

Cet essai compare systématiquement tous les motifs communs qu'on trouve chez Pétrone et les romans grecs d'amour (plus récents que lui) qui nous sont parvenus, pour vérifier si des thèmes employés sérieusement par les auteurs grecs sont traités de manière ridicule ou parodique chez Pétrone. Cette hypothèse est vérifiée presque sans exception. À la fin de la recherche on sera en mesure de juger si cela est dû simplement au pur hasard et, s'il y a un rapport entre les écrivains grecs et Pétrone, si l'emploi sérieux a ou non précédé la parodie. Dans l'un comme dans l'autre cas la réponse nous semble n'admettre aucun doute raisonnable. Pétrone a donc parodié une tradition plus ancienne que les romans grecs que nous lisons encore.

This paper systematically compares all the common motifs that appear in Petronius and in the (later) Greek love romances that have come down to us, in order to ascertain whether certain themes seriously employed by the Greek authors receive a ridiculous or parodic twist in Petronius. This appears to be the case almost with no exception. At the end of the inquiry we shall be in a position to judge whether this is due to mere hazard, and, if there is a connection between the Greek writers and Petronius, whether or not serious use has preceded parody. For both questions, there is apparently no doubt. The butt of Petronius' parody, therefore, must be a novelistic tradition more ancient than the romances we can still read. 
INDEX

Mots-clés : Apulée, Lucien, parodie, Pétrone, Roman grec, Satyricon

\section{AUTEUR}

ALDO SETAIOLI

Université de Pérouse 